reliable) method of examining sinuses.' He can so help to exclude this commonly unsuspected cause of rheumatism, eye-trouble, headache and chronic dyspepsia, and may thus obviate an unnecessary visit to a rhinologist.

Another way in which they help is in the demonstration of the presence of polypi (and therefore of latent infection) in the antral lining. For some unexplained reason, many antral polypi which are quite translucent in the dark room show up very clearly by. X-rays.

In conclusion, it should be realised that while the complete cure of a chronic sinusitis may call for operative measures, yet much relief may usually be afforded by less drastic means; and that in acute sinusitis, with the exception of really fulminating cases, non-operative means should be the method of choice. The intelligent use of cocaine is the greatest stand-by at our command in acute disease of the nose, the drug preferably being "protected" by some adjuvant vaso-constrictor such as ephedrine ; and there would be fewer cases of chronic sinusitis if the profession would realise what a potent weapon we possess in cocaine for the relief of obstruction both to the airway and to the exits from the sinuses.

In chronic sinusitis, effective measures of a non-operative nature are harder to come by: but it should be recognized that the routine use of nasal lotions may be largely stultified by failure to ensure that the lotions attain to the chinks and crannies for which they are intended; the desired end may be furthered by occasional shrinking of the congested nasal mucus by vaso-constrictors, as a prelude to the sniffing or spraying of the usual alkaline nose-wash. Another quite potent weapon is the intramuscular injection of collosol manganese, which, in a fairly well-ventilated nose, is especially likely to help in clearing up the low-grade catarrh which so often follows influenza and for which vaccines are often ineffective.

\title{
THE SIGNIFICANCE OF BLEEDING AS A SYMPTOM IN GYN ECOLOGY.
}

\section{BY MARTIN OLDERSHAW, M.D.LOND., F.R.C.S.ENG.,}

Hon. Surgeon to In-and Out-patients, the Hospital for Women, Soho Square, W.1.

IN general practice, gynæcological conditions form a large and important group of cases. These patients are often reluctant to seek advice unless some urgent symptom is present, and in many cases the symptom which first sends them to a doctor is vaginal bleeding. Such bleeding may be due to a large number of causes, many of them trivial, others extremely serious, and in this lecture I have attempted to outline the various conditions which may be present, and to describe the way in which the problem of diagnosis may be tackled.

It is convenient to arrange cases into three groups according to age, namely, "Before Puberty," " During Reproductive Life," and "After the Menopause."

Before Puberty.-Vaginal bleeding is rare in children and is usually due to trauma, such as scratching, attempted rape, or the insertion of a foreign body, for example, a pencil. If bleeding occurs in the absence of any injury, thorough examination is required,

${ }^{1}$ Hodgson, British Medical Journal, January 7, 1933. 
for which an anæsthetic is usually necessary. Possible causes are : grape-like sarcoma of the cervix ; angioma of the vagina ; and occasional virulent infection of the vulval region, e.g., in diphtheria.

During Reproductive Life.-The period from puberty to the menopause, or roughly from 15 to 50 years, furnishes the great majority of cases. Here the first question to be settled is, whether the bleeding is due to some complication of a present pregnancy or following a recent miscarriage or labour.

\section{Bleeding Connected with Pregnancy.}

Sometimes the relation is obvious enough, but often careful examination of the menstrual history is required. It is important to ask if any period was missed, or was at all late before the present trouble started. If the answer is affirmative in a woman who is normally regular it is strongly suggestive of pregnancy. In such a case, the next step is to determine whether the pregnancy is still continuing, or whether miscarriage has already occurred or is imminent.

If, on bi-manual examination, signs of an early pregnancy are found and the cervix $\bigcirc$ is closed, the condition is one of threatened abortion, and should be treated by rest and sedatives. If the internal os is dilated, the abortion is inevitable, and may be assisted by full doses of ergot or, should hæmorrhage be excessive, by immediate evacuation of the uterus under an anæsthetic.

If the uterus is somewhat bulky, but has lost the globular shape characteristic of early pregnancy, and the cervix is dilated, an early miscarriage has occurred recentl Such cases usually clear up with ergot, but in deciding treatment the consistence of the uterus is more important than its size. An empty uterus is firm, and the cervix soon contracts. A uterus containing retained products is soft and flabby, and such a uterus usually needs curetting.

Moles are rarely seen because the common or blood-mole seldom causes enough symptoms to send the patient to a doctor. The rare vesicular mole is more serious. The only certain method of diagnosis is to see or feel a vesicle, but it may be suspected if the uterus is unduly large for the period of amenorrhœa and a blood-stained watery discharge is present. The uterus should be emptied as soon as the condition is diagnosed, preferably by inserting a tent, and a close watch kept during the ensuing three months for the possible development of a chorion-epithelioma. Should any irregular bleeding $₹$ occur during this time, exploratory curetting should be done and the fragments removed $\cong$ and examined microscopically.

Another possible cause of bleeding in early pregnancy is ectopic gestation. This dangerous condition is generally easy to diagnose, the combination of pain, slight vaginal $\tilde{\sigma}$ loss, and the signs of severe internal hæmorrhage being distinctive. Difficulty may arise ${ }_{0}$ in less urgent cases, but while in an ordinary miscarriage the patient complains of $\tilde{N}$ "bleeding" with pain as a minor symptom, in ectopic pregnancy she complains of "pain" with bleeding as a minor symptom.

Bleeding after the twenty-eighth week of pregnancy, or within a month after labour, $\stackrel{\Phi}{\stackrel{\Phi}{.}}$ is an obstetric problem, and will not be discussed here.

Menorrhagia commencing as soon as the periods become established after labour $\frac{\vec{D}}{\mathbb{D}}$ suggests subinvolution, possibly due to sepsis or retroversion. Irregular bleeding $\frac{0}{\infty}$ 
starting within a few months of labour is not common, and may be due to another early pregnancy, or possibly to chorion-epithelioma.

\section{Bleeding apart from Pregnancy.}

This large group includes the majority of cases seen. Here the first point to be settled is whether the bleeding complained of occurs solely at the period, i.e., as a pure menorrhagia, or whether it occurs independently of these, either at irregular intervals or more or less continuously.

The following are common causes of menorrhagia: (I) Endocrine disturbance; (2) pelvic infections ; (3) fibroids or other simple tumours ; (4) retroversion.

(I) Endocrine disturbance sufficiently marked to affect menstruation is fairly common around puberty and the menopause, but rare during the middle of reproductive life. The onset and cessation of full physiological activity of the ovaries throws considerable strain on the smooth working of the endocrine system, and it is not surprising that sometimes the mechanism breaks down. A typical instance of a purely "endocrine" menorrhagia is the menorrhagia of puberty. Mild cases of this condition are fairly common, and usually undergo spontaneous cure in a short time. Severe cases are difficult to deal with. It is justifiable to curette once in order to make a complete diagnosis, but the curative effect is nil, and drugs often fail. Ergot is useless, but calcium lactate, $30 \mathrm{gr}$., on alternate nights, and potassium, chlorate 5 gr., t.i.d., is worth trying for two months. In severe cases the basal metabolic rate should be estimated. It is often raised, indicating hyperthyroidism, if so, X-ray treatment of the thyroid may greatly lessen the periods.

Another example of "endocrine menorrhagia" is the occasional flooding occurring in a patient near the menopause, with apparently normal pelvic organs. At this age, however, the greatest care is needed to exclude any local disease in the uterus.

(2) Pelvic infections, however caused, produce the typical syndrome of menorrhagia, leucorrhwea, and backache. There are two main groups of cases : (a) Septic infection following labour or miscarriage ; $(b)$ gonorrhœal, often chronic, with a secondary septic infection added, usually associated with sterility.

(3) Uterine fibroids are the commonest benign tumours, and cause more or less severe menorrhagia whenever they become submucous. The size of the fibroid bears no relation to the degree of bleeding; a large sub-peritoneal fibroid filling the pelvis may cause none at all, while a fibroid polypus too small to be felt bi-manually may cause seriously profuse periods.

Ovarian tumours have little direct effect on menstruation, but may produce slight menorrhagia owing to general pelvic congestion caused by the tumour.

(4) Retroversion will never by itself cause menorrhagia. If, however, any other cause be present, such as chronic infection, or mild endocrine disturbance, any retroversion will undoubtedly make the menorrhagia worse.

\section{Bleeding not connected with Pregnancy or Menstruation.}

Uterine bleeding unconnected with pregnancy or menstruation is comparatively rare, but it is a very important symptom which should at once arouse suspicion of definite organic disease. Care is needed not to place bleeding due to a miscarriage in this group. The patient may be ignorant that she is pregnant, or may suppress the fact. 
In women under 35 years the only common causes of more or less persistent ̊ bleeding are : (I) Simple polypus of the uterus; (2) cervical erosion. In older women $\frac{\bar{D}}{\mathrm{Q}}$ the possibility of malignant disease must always be borne in mind.

The sole early symptom of uterine cancer is irregular bleeding or a blood-stained discharge, such bleeding being quite independent of the periods, or commencing after $\stackrel{0}{9}$ the menopause. Whenever this symptom is present in a woman over 35 , the only safe $\frac{\bar{C}}{\sigma}$ working rule is to assume that the case is malignant until proved otherwise. It is often $\frac{\overline{\bar{N}}}{\frac{\bar{\sigma}}{\bar{S}}}$ difficult to obtain a reliable history. Remember that near the menopause an isolated $\stackrel{\mathbb{\Phi}}{\Omega}$ severe flooding, which at once sends the patient to her doctor, may be simply due to endocrine disturbance, while a quite trivial coloured discharge which is only too easily $\vec{\circ}$ ignored is the typical early sign of cancer. It is important to ask if there is any bleeding, $\overrightarrow{\vec{\omega}}$ however slight, in between the periods, or for example after douching. If such bleeding $\stackrel{\circ}{\circ}$ does occur a complete pelvic examination is essential.

Carcinoma of the cervix occurs typically in multiparæ, aged about 45 . In such $\frac{3}{6}$. patients the cervix is often torn, hard and bulky, owing to the stress of past labours, but $\varnothing_{0}^{\circ}$ if the surface is smooth, and does not bleed, no growth is present. The important point o is to feel for any rough patch which is friable when scratched with the finger-tip and $\mathrm{O}$ bleeds when this is done. Such a patch is almost certainly carcinomatous, but if in any doubt a small piece should be excised for microscopic examination.

Cancer of the body is less easy to detect. It is much less common, usually occurs $\frac{\widehat{O}}{د}$ after the menopause at about 55 years and is as common in nulliparæ as multiparæ. $\vec{\omega}$ The only early symptom is slight irregular bleeding. On bimanual examination the cervix and whole uterus may feel perfectly normal, but that important danger signa毒 a spot of blood, is left on the examining finger. Exploratory curetting should be done without delay. This is the only possible method of diagnosis in early cases, which have an excellent chance of permanent cure by operation, the prognosis being much better than with cancer of the cervix.

Other causes of irregular bleeding are rare, and generally.occur after the menopause so they will be described under this heading.

\section{Bleeding after the Menopause.}

A majority of cases with this symptom have cancer of the uterus, and therefore need prompt and thorough investigation on the lines just described, but occasionally the bleeding may be due to one of the following conditions : (I) Infected polypus; (2) 을 neglected pessary ; (3) senile endometritis ; (4) cervicitis and urethral caruncle.

(I) A large gangrenous fibroid polypus, or (2) a grossly neglected pessary, may cause $D$ symptoms exactly like an advanced cancer of the cervix. The diagnosis is obvious in both cases. (3) Senile endometritis. This is not very common. A thin purulent $\tilde{N}$ discharge is present with only a few spots of blood. The uterus is very small and $\mathbb{O}$ atrophic. (4) Chronic cervicitis and urethral caruncle. The discharge comes from the infected cervix and the slight bleeding from the caruncle. Examination reveals the caruncle, and the fact that no uterine bleeding is present.

Summary.-The following scheme recapitulates the chief points in diagnosing any $\stackrel{\mathbb{D}}{\mathscr{D}}$ given case of bleeding during Reproductive life.

(I) A complication of pregnancy.

(2) Unconnected with pregnancy. 
If a pure menorrhagia commonly due to :-

(i) Endocrine disturbance.

(ii) Pelvic infections.

(iii) Fibroids or other simple tumours.

(iv) Retroversion.

If independent of the periods.

Under 35 suspect polypus.

Over 35 suspect uterine cancer.

In conclusion attention must again be drawn to the fact that slight irregular bleeding is the only early symptom of uterine cancer. It is, therefore, of the greatest importance, not only that every patient presenting this symptom should be thoroughly examined, but no less so that careful inquiry as to the presence or absence of such irregular bleeding should be made.

\section{SOME AFFECTIONS OF THE SKIN IN CHILDHOOD.}

\section{BY GODFREY W. BAMBER, B.A., M.D., M.R.C.P., \\ Physician to the Skin Department, Victoria Hospital for Children, Miller General Hospital and Metropolitan Hospital.}

THE general practitioner with a limited knowledge of skin diseases, who is astonished and confused hy the number of names and descriptions which he finds on consulting a textbook of dermatology, will be relieved to hear that in children the variety of common skin complaints is less than in adults, and that once the correct diagnosis has been made almost all of these conditions are amenable to treatment.

It is convenient to deal with congenital abnormalities first, because children are brought in earliest infancy to see the doctor on account of a very common congenital malformation of the skin, a nævus, or to adopt dermatological nomenclature, an hæmangioma. This developmental defect may appear either as the "strawberry mark" with a slightly raised, irregular, bright red surface, or as a flat patch, the "port wine stain " in which the intensity of the colour depends on the number and depth of the dilated capillaries, or lastly as the subcutaneous hæmangioma, a spongelike tumour from which the blood can easily be expressed. An angioma may conform to one or may be made up of all three types. Parents and relatives are apt to view these marks with apprehension, although there is no necessity for alarm; and consciously or unconsciously influenced by the fear that something must be done urgently, the doctor carries out treatment which in many cases is far too precipitate and vigorous. Angiomata, apart from the port-wine stain, are rarely found in adults, nor do many of these show scars resulting from excessive treatment or even excision. The natural history of angiomata is that they are present at or appear soon after birth ; their rate of growth is for a time more rapid than that of the baby ; then they involute slowly, and often disappear without a trace of their former presence. A good illustration of this is seen on the neck. Every third baby has a flat capillary angioma below the occiput. The colour varies from pale pink to dark red. In a few months most of these marks have gone, although a few, generally the darker ones, may be found when examining the hair of adults. These marks have been said to result from intra-uterine trauma, and 